Check for updates

Cite this: RSC Adv., 2018, 8, 21229

\title{
Enhancing the anti-ovarian cancer activity of quercetin using a self-assembling micelle and thermosensitive hydrogel drug delivery system
}

\author{
Guangya $\mathrm{Xu}^{\mathrm{a}}{ }^{\mathrm{B}}$ Bin Li, ${ }^{\mathrm{a}}$ Ting Wang, ${ }^{\mathrm{a}}$ Jun Wan, ${ }^{\mathrm{a}}$ Yan Zhang, ${ }^{\mathrm{a}}$ Jingwei Huang ${ }^{\mathrm{a}}$ \\ and Yangmei Shen (D) *bc
}

Ovarian cancer, as one of the killers that threaten women's health, has been studied extensively. As a natural bioflavonoid with prospective effects, quercetin is highly recognized for its anti-cancer applications. However, one of the major challenges that quercetin faces is its poor water solubility, instability in physiological media, and subsequent poor bioavailability. Thus, optimizing the ideal drug delivery options is necessary to facilitate the harnessing of the maximum benefits from quercetin. In this study, a quercetin-loaded thermosensitive injectable hydrogel system (Qu-M-hydrogel composites) was constructed based on nanotechnology. Quercetin was encapsulated into MPEG-PCL (with a high drug loading of $7 \%$ and minor particle size of $32 \mathrm{~nm}$ ) and then added into the blank thermosensitive hydrogel Pluronic F-127. The Qu-M-hydrogel composites showed a much slower release than Qu-M in vivo. Moreover, the cytotoxicity, apoptosis induction, and anti-tumor effects of the Qu-M-hydrogel composites on the abdominal SKOV-3 ovarian cancer mouse models were investigated in vivo. Compared with other groups, the Qu-M-hydrogel composites exhibited improved apoptosis induction and cell growth inhibition effects and in vivo trials showed a better balance between the anti-tumor efficacy in the Qu-M-hydrogel composite group than in other groups at an equal drug dose. In conclusion, the prepared Qu-M-hydrogel composites enhanced the anti-tumor activity by providing a high local quercetin concentration, sustained and stable drug release, extended drug retention inside the tumor, and low toxicity to normal tissues. The Qu-M-hydrogel composites might have great potential for clinical application in anti-ovarian cancer activity.

Received 17th April 2018
Accepted 17th May 2018
DOI: $10.1039 /$ c8ra03274b
rsc.li/rsc-advances

\section{Introduction}

As one of the top ten common causes of cancer deaths in women, ovarian cancer is among the most familiar malignant tumors in female reproductive organs, ranking seventh in female tumors. A recent finding has provided the first concrete clue that 240000 females are diagnosed with ovarian cancer worldwide every year, with an overall 5 year survival frequency of less than $50 \%$, leading to approximately 15000 deaths per year. ${ }^{1,2}$ It is estimated that there will be 282741 new cases diagnosed with ovarian cancer worldwide by 2020 , and about $66 \%$ of them will be women under 65 years of age..$^{3-5}$

\footnotetext{
${ }^{a}$ Department of Anatomical Pathology and Pathophysiology, College of Medicine, Chengdu University, Chengdu, People's Republic of China

${ }^{b}$ Department of Pathology, West China Second University Hospital, Sichuan University, Chengdu, 610041, PR China. E-mail: symjulia@126.com; Fax: +86 2885164060; Tel: $+862885164063$

'Key Laboratory of Birth Defects and Related Diseases of Women and Children (Sichuan University), Ministry of Education, West China Second University Hospital, Sichuan University, Chengdu, 610041, PR China
}

The development of ovarian cancer is influenced by various risk factors, such as reproductive factors, exogenous hormones, genetics, and lifestyle. Age, family history of ovarian cancer, infertility, environmental ionizing radiation, and asbestos are key known factors that cause this cancer, and there is limited evidence that the personal use of pesticides by women also has a relationship with this cancer. ${ }^{6,7}$ At present, the primary treatments of ovarian cancer are cytoreductive surgery and platinum-based chemotherapies, but the prognosis is still poor because the confirmed diagnosis is usually at a terminal stage..$^{8,9}$ So, new and potentially effective treatments for ovarian cancer are in urgent demand.

Quercetin, as the most typical flavonoid, is an important kind of metabolite from plants, which is present in many foodstuffs, fruits, vegetables, rhizomes, tree barks, flowers, and tea in bound (flavonoid glycosides) or free state (flavonoid aglycones) forms. ${ }^{10-12}$ It is well known for its DNA protection, anti-inflammatory, antiviral, and antibacterial properties, etc. ${ }^{13,14}$ Research suggests that eating more fruits and vegetables containing flavonols and flavanones can reduce the risk of ovarian cancer. The latest research demonstrates that quercetin has a variety of pharmacological activities and its anti-cancer 
activity can induce tumor cell apoptosis, regulate tumor cell proliferation and tumor angiogenesis, and inhibit the signal pathways of many important targets. ${ }^{15-24}$ All the advantages make quercetin nature's nominee for a cancer cure. However, quercetin's low water solubility is a fatal weakness that limits its effectiveness in clinical application significantly. In current literature, its role in curing ovarian cancer is rarely recorded. Therefore, it is valuable to explore an efficient delivery system for quercetin to dissolve smoothly and continuously, thereby allowing it to act on ovarian carcinoma sustainedly.

Chemotherapy is a routine treatment for peritoneal carcinomatosis, and traditional intravenous chemotherapy has severe toxic side effects, including immunosuppression, neurotoxicity, and myelosuppression. Those side effects reduce the strength of the chemotherapy and bring great pain to the patients. $^{25-29}$ How to reduce the toxic injury and maintain effective drug concentrations is still a thorny problem for doctors. As a promising strategy, localized drug delivery technology has been studied for many years, which can not only reduce the side effects on normal tissues and avoid the systemic circulation of chemotherapeutic drugs, but also provide the localized sustained release of chemotherapeutic drugs and thereby enhance the therapeutic effect. ${ }^{30-32}$

Intraperitoneal chemotherapy, as one of the targeted approaches, can keep local drug concentrations at an effective level and, at the same time, reduce the systemic exposure of the chemotherapeutic drugs. ${ }^{33,34}$ However, conventional intraperitoneal chemotherapy drugs have a short residence time and require frequent or continuous dosing. How to keep the effective drug concentration to the regional malignancies during the protracted duration of intraperitoneal chemotherapy is challenging. To overcome this technical problem, some new drug delivery systems, including nanoparticles, liposomes, and microspheres, have been developed to maintain drug concentrations, extend the duration time, and maximise reduction of the systemic toxicity. However, the controllability of the drug release of all those methods is restricted, and their larger particles may induce the inflammatory reactions and other problems..$^{35,36}$

Biodegradable thermosensitive hydrogels, with the specific characteristic that they can convert between solution and gel state, have been explored as a promising strategy for localized implantable systems. ${ }^{37,38}$ Thermosensitive hydrogels have a low critical solution temperature that can carry drugs to a predetermined site in a liquid state and form a crosslinked hydrogel at physiological temperature. ${ }^{39}$ The potential application prospects of thermosensitive hydrogels show a satisfactory solution for sustained drug delivery and reduction of drug resistance, thereby making them popular in local drug delivery systems. Anti-cancer drugs carried by thermosensitive hydrogels usually release smoothly and steadily, thereby lengthening drug duration time and reducing side effects. ${ }^{40}$ Ovarian cancer is a peritoneal disease, with peritoneal fluid as a carrier to promote the spread of tumor cells. Intraperitoneal chemotherapy with thermosensitive hydrogels may be a better choice for treating ovarian cancer.

Aiming at improving the application of quercetin and searching for a new way to treat ovarian cancer, an injectable thermosensitive hydrogel system with quercetin loaded was developed in this study. Quercetin, wrapped in double layers (MPEG-PCL and hydrogel), was comprehensively tested for its anti-tumor effects on ovarian cancer. Cell viability and inducedapoptosis tests were carried out in parallel and, at the same time, a tumor-bearing mouse model was established to observe cell growth inhibition. Histological analyses were performed to detect the variation of cancer cells: MTT for cytotoxicity, TUNEL analysis for apoptosis, CD31 analysis for anti-angiogenesis, and Ki67 analysis for anti-proliferation. The results showed that the approach of double encapsulation by MPEG-PCL and hydrogel enhanced the anti-tumor activity of quercetin.

\section{Materials and methods}

\subsection{Materials}

Quercetin, Dulbecco's Modified Eagle's Medium (DMEM), and 3-(4,5-dimethylthiazol-2-yl)2,5-diphenyltetrazo-lium bromide (MTT) were provided by Sigma-Aldrich Chemical Co (U.S.). Pluronic F-127 was kindly supplied by BASF Ltd (Shanghai, China) and acetone, ethanol, methanol, dimethyl sulfoxide (DMSO), methylene chloride, and HPLC grade acetic acid were obtained from Kelong Chemical Co (China). Nude female BALB/ c mice that were 6-8 weeks-old were acquired from the Laboratory Animal Center of Sichuan University (China). All animal procedures were approved and controlled by the Institutional Animal Care and Treatment Committee of Sichuan University and carried out according to the Animal Care and Use Guidelines of Sichuan University.

\subsection{Preparation of Qu-M and the Qu-M-hydrogel composites}

MPEG-PCL was prepared according to previously published methods and quercetin and MPG-PCL polymeric nanomicelles (Qu-M, QM) were prepared in a self-assembly method. In brief, $93 \mathrm{mg}$ of MPEG-PCL and $7 \mathrm{mg}$ of quercetin were dissolved in $10 \mathrm{~mL}$ of acetone as the organic phase. The organic solvent was then removed by vacuum rotary evaporation at $55{ }^{\circ} \mathrm{C}$ to form a film. Finally, a normal salt solution was added to dissolve the film and QM was collected. The blank nanomicelles were prepared the same way without quercetin. All the particles were then lyophilized and stored at $4{ }^{\circ} \mathrm{C}$.

$15 \mathrm{mg}$ of Pluronic F-127 powder was dissolved in $50 \mathrm{~mL}$ of PBS solution and then magnetically stirred for 24 hours in an ice bath to allow Pluronic to be sufficiently dissolved in PBS. The mixture was sterilized under high temperature and pressure conditions for $20 \mathrm{~min}$ and cooled to $4{ }^{\circ} \mathrm{C}$ to form a sol. The premade QM were then mixed with Pluronic F-127 hydrogel to form a homogeneous solution, and the concentration of the Qu-Mhydrogel composites (QMGs) was kept at $20 \mathrm{wt} \%$. The empty hydrogel was prepared by the same method without quercetin.

\subsection{Characterization of Qu-M and the Qu-M-hydrogel composites}

In order to observe the morphology of the Qu-M (QM), the samples were dissolved in distilled water and then mixed with an equal amount of $2 \%$ aqueous sodium phosphotungstate 
solution. Afterwards, the mixed liquor was pipetted on a nitrocellulose-coated copper grid, dried at room temperature negatively, and then the stained specimens were examined by transmission electron microscopy (TEM) (H-6009IV, Hitachi, Japan). The particle size of the Qu-M (QM) was analysed using dynamic light scattering (Zetasizer Nano Series-ZS90, Malvern Instruments) at $25^{\circ} \mathrm{C}$, as well as the zeta potential.

High performance liquid chromatography (HPLC) was employed to determine the drug loading (DL) and encapsulation efficiency (EE) of Qu-M (QM). Briefly, $5.0 \mathrm{mg}$ of lyophilized Qu-M (QM) was dissolved in $0.2 \mathrm{~mL}$ of dichloromethane (DCM) and diluted with $0.8 \mathrm{~mL}$ of methanol, and then once it had fully dissolved, the mixture was centrifuged at $12000 \mathrm{rpm}$ for 20 minutes at room temperature. The obtained supernatant was determined by High Performance Liquid Chromatography (HPLC, LC-20AD, Shimadzu Co, Ltd) and the drug loading (DL) and encapsulation efficiency (EE) were calculated according to eqn (1) and (2).

$$
\begin{gathered}
\text { Drug loading }=\frac{\text { drug }}{\text { polymer }+ \text { drug }} \times 100 \% \\
\text { Encapsulation efficiency }=\frac{\text { experimental drug loading }}{\text { theoretical drug loading }} \times 100 \%
\end{gathered}
$$

Qu-M hydrogel (5 mg of Qu within $1 \mathrm{~mL}$ of F127 hydrogel) was introduced in glass bottles containing $10 \mathrm{~mL}$ of $0.5 \%$ Tween-80 in PBS $(\mathrm{pH}=7.4) .100 \mu \mathrm{L}$ of the PBS in the bottles was replaced at specified intervals. The drug concentrations in PBS were determined using a high performance liquid chromatography instrument (Waters Alliance 2695). The UV absorbency was monitored at $421 \mathrm{~nm}$ using a C18 column (Grace analysis column).

\subsection{In vitro cytotoxicity assay}

The cytotoxic effects of free quercetin and Qu-M (QM) on ovarian cancer SK-OV-3 cells in vitro were evaluated by MTT colorimetric assay. SKOV-3 cells were sowed into 96-well plates at a density of $5 \times 10^{3}$ per well and incubated for 24 hours with Dulbecco modified Eagle's medium (DMEM) containing 10\% fetal bovine serum (FBS). The cells were washed once with DMEM medium and co-incubated with Qu-M (QM) and free quercetin $(\mathrm{FQ})$ at different quercetin concentrations $(0.125 \mu \mathrm{g}$ $\mathrm{mL}^{-1}, 0.25 \mu \mathrm{g} \mathrm{mL} \mathrm{mL}^{-1}, 0.5 \mu \mathrm{g} \mathrm{mL}^{-1}, 1 \mu \mathrm{g} \mathrm{mL} L^{-1}, 2 \mu \mathrm{g} \mathrm{mL} \mathrm{g}^{-1}, 4 \mu \mathrm{g}$ $\mathrm{mL}^{-1}, 8 \mu \mathrm{g} \mathrm{mL} L^{-1}, 16 \mu \mathrm{g} \mathrm{mL} L^{-1}$, and $32 \mu \mathrm{g} \mathrm{mL}^{-1}$ ). Then the cell viability was measured by MTT colorimetric assay 24 and 48 hours after co-incubation.

As a widely used method for the detection of cell activity, MTT is one kind of yellow dye with the scientific name of 3-(4,5dimethylthiazole-2)-2,5-diphenyltetrazole bromide and trade name of thiazole blue. The succinate dehydrogenase in the mitochondria of viable cells can reduce exogenous MTT to waterinsoluble blue-violet crystals (formazan) and deposit them in the cells. Within a certain number of cells, the amount of MTT crystals formed is proportional to the number of cells, while dead cells do not possess this function. Formazan crystals could be dissolved by dimethyl sulfoxide (DMSO), showing blue-violet color. Then the absorbance of the colored solution quantified by the plate reader (OPTImax, Molecular Dynamics, CA, USA) at a wavelength of $570 \mathrm{~nm}$ can reflect the number of viable cells indirectly. All results were compared to the untreated cells as $100 \%$ survival, and the cells' viability was expressed as a percentage.

\subsection{In vitro cell apoptosis assay}

The apoptosis effects of Qu-M (QM) on ovarian cancer SKOV-3 cells in vitro were assessed by flow cytometry. First, the washed SKOV-3 cells were incubated with Qu-M (QM) for 48 hours. The concentrations of quercetin in the groups were $0 \mu \mathrm{g}$ $\mathrm{mL}^{-1}, 4 \mu \mathrm{g} \mathrm{mL} \mathrm{m}^{-1}, 8 \mu \mathrm{g} \mathrm{mL} L^{-1}, 16 \mu \mathrm{g} \mathrm{mL} L^{-1}, 32 \mu \mathrm{g} \mathrm{mL} L^{-1}$ and $64 \mu \mathrm{g}$ $\mathrm{mL}^{-1}$. Second, the obtained cells were double stained by AnnexinV and propidium iodide (PI), and then detected by flow cytometry (FCM, ESP Elite, Beckman-Coulter, Miami, FL). The proportion of the Annexin-V-positive cells representing early apoptosis, as well as the percentage of PI-positive indicating late apoptosis, were added up to express the apoptosis rates.

\subsection{In vivo cancer model}

For the purpose of establishing the animal model of ovarian cancer, healthy female nude BALB/c mice aged 6-8 weeks under standard conditions in the animal research center of Sichuan University were employed, consuming a normal diet and acidified water without antibiotics. The SKOV-3 cells $\left(3 \times 10^{6}\right)$ were inoculated intraperitoneally into the abdominal cavity of the healthy nude female BALB/c mice aged 6-8 weeks.

After 7 days, when the mice's subcutaneous tumors grew to an average diameter of about $6 \mathrm{~mm}$, all of the mice were randomly divided into four groups with 5 or 6 mice each: Qu-M-hydrogel composites (QMGs), Qu-M (QM), free quercetin (FQ), empty hydrogel (EG), and normal saline (NS) groups. With the exception that the preparations of free quercetin $(\mathrm{FQ})$ were mixed with polysorbate-80 (Tween-80) and free ethanol $(1: 1, \mathrm{v} / \mathrm{v})$, the other group preparations were all in physiological saline. Then each mouse in the treated groups was administered a dose of $50 \mathrm{mg}$ $\mathrm{kg}^{-1}$ (quercetin) via intraperitoneal injection once every 3 days for 24 consecutive days. When the control animals began to decrease, following sacrifice by cervical spine dislocation, the tumors of all the mice were harvested and examined immediately with regards to the tumor weight and tumor nodule.

\subsection{CD31 assay}

The immunofluorescence analysis of neovascularization (CD31) was used to detect the anti-angiogenesis activities of the Qu-Mhydrogel composites (QMGs), Qu-M (QM), free quercetin (FQ), empty hydrogel (EG), and normal saline (NS) in the tumor tissues of the animal models. CD31 was used primarily to demonstrate the presence of the endothelial cell tissue in the immunohistochemistry and thereby to evaluate the tumor angiogenesis. First, the tumor tissues obtained from the animal models were quickly put into liquid nitrogen to freeze, and dissected into $5 \mu \mathrm{m}$ sections. Then the slices, which were kept at room temperature for 30 minutes, were fixed in acetone at $4{ }^{\circ} \mathrm{C}$ 
for several minutes, and then washed with phosphate buffer (PBS, $\mathrm{pH}=7.4$ ). Second, the sections were stained with the rat anti-mouse CD31 polyclonal antibody and rhodamineconjugated secondary antibody sequentially on the basis of the manufacturer's instructions. Third, all the samples were observed with the preceding procedures and the microvessel densities (MVD) was expressed as the number of microvessels, which were quantified by fluorescence microscopy in highpower $(\times 40)$ microscopic fields.

\subsection{Ki-67 assay}

Immunofluorescence technology was employed to determine the Ki-67 protein in the tumor tissues for estimating the antiproliferation effects of the Qu-M-hydrogel composites (QMGs), Qu-M (QM), free quercetin (FQ), empty hydrogel (EG), and normal saline (NS) in vivo. Generally speaking, all methods and steps were the same as the "CD31 assay", whereas the differences were that the Ki67 protein shows green fluorescence, and the nucleus displays blue fluorescence. The cell proliferation rate was expressed as the percentages of the Ki-67 positive cells and total cells.

\subsection{Tunnel assay}

The induced-apoptosis properties of the Qu-M-hydrogel composites (QMGs), Qu-M (QM) and free quercetin (FQ) in vivo were determined by tunnel analysis and compared with the groups of empty hydrogel (EG) and normal saline (NS). The tumor tissues harvested from the animal models were fixed in PBS containing $4 \%$ paraformaldehyde for 24 hours and rinsed with phosphate buffer (PBS, $\mathrm{pH}=7.4)$. Then, they were immersed in different concentrations of ethanol solution for gradient dehydration, the use of xylene transparent, and embedded into paraffin wax. Subsequently, the wax was cut to a thickness of 3-5 $\mu \mathrm{m}$ to be checked.

After pretreatment, the tumor slices were stained according to the relevant instructions of the TUNEL assay (Promega Co, Madison, Wisconsin, USA), and observed by fluorescence microscopy. Apoptotic cells showed green fluorescence at a wavelength of $520 \pm 20 \mathrm{~nm}$ and 5 pictures $(40 \times)$ of them were taken stochasticly and analyzed by microscopic image analysis software (Axios Vision Rel4.8, Carl Zeiss Meditec AG).

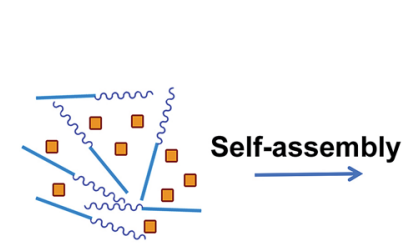

Qu and MPEG-PCL

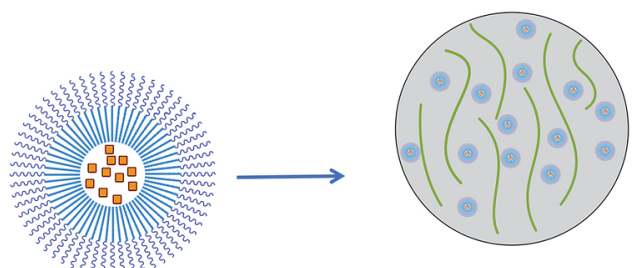

Qu nano-micelles
Qu nano-micelles-hydrogel

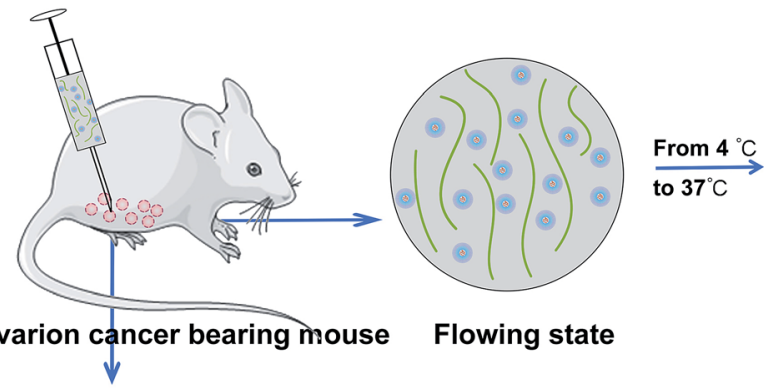

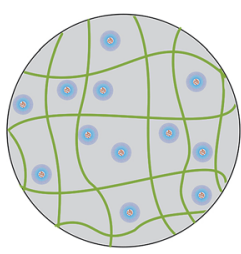

Gel state
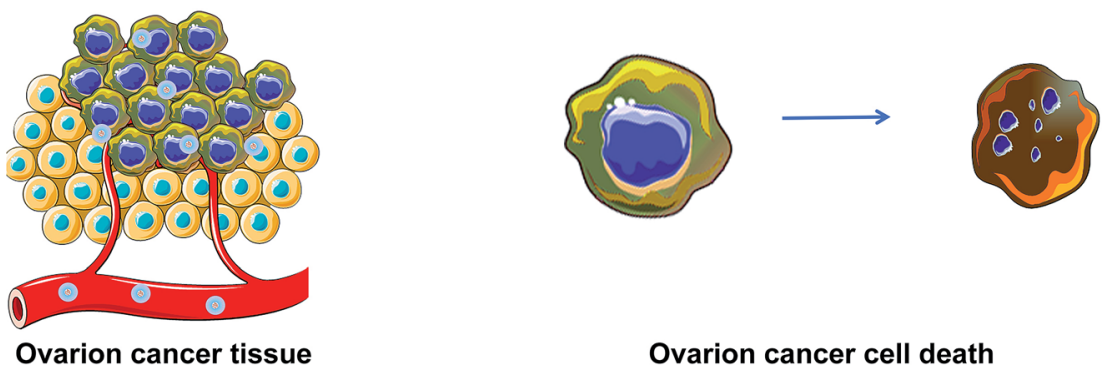

Ovarion cancer cell death

Fig. 1 The preparation scheme of the Qu-M and Qu-M-hydrogel. Qu-M (QM) was prepared with a self-assembly method. Firstly, quercetin and MPEG-PCL were co-dissolved in the acetone solution. In this process, quercetin and MPEG-PCL were assembled automatically into Qu-M (QM). Then, the Qu-nano-micelles was added to the hydrogel at $4{ }^{\circ} \mathrm{C}$, and Qu-M dispersed to form a hydrogel. When the Qu-M-hydrogel composites (QMGs) were injected in to the abdominal cavity of the ovarian tumor bearing mice, the hydrogel changed from flow state to a gel state. The quercetin was released slowly near the tumor tissue from the Qu-M-hydrogel composites (QMGs). 


\subsection{Safety evaluation of Qu-M and the Qu-M-hydrogel composites}

The tumor-bearing mouse models were observed continuously for 31 days, including the general conditions (the activity, hair, faeces, behavior pattern, body weight, etc.), and mortality. At predetermined days, the rats were sacrificed, and the dorsal region was sectioned. After sacrifice, blood was collected for the detection of the hematologic toxicity and the main organs of the hearts, livers, spleens, lungs, and kidneys were collected for histopathological studies. All samples were fixed in $10 \%$ neutral-buffered formalin and embedded in paraffin, then the tissues were sectioned and stained with hematoxylin and eosin (H\&E). The histological changes were evaluated through observation under a light microscope.

\subsection{Statistical analysis}

Where appropriate, all the results were obtained from triplicate experiments performed in a parallel manner, and depicted as the mean \pm SD. The tumor volumes and weights of each group were compared by one-way ANOVA, and the survival curves were estimated according to the Kaplan-Meier method. The levels of the $P$ values for comparison between all groups were determined by a 2-tailed Mann-Whitney test, and a probability value of $\leq 0.05$ was considered to be statistically significant. Statistical analyses were performed with the statistical software system of SPSS v19.0 for windows (IBM, Armonk, NY).

\section{Results}

\subsection{Synthesis and characterization of Qu-M and the Qu-M-} hydrogel composites

3.1.1 Self-assembly of Qu-M and the Qu-M-hydrogel composites. In order to improve the low water solubility of quercetin and increase its bioavailability, the amphiphilic block copolymer (MPEG-PCL) was used to prepare the quercetin nanomicelles in this study. Quercetin was encapsulated
A)

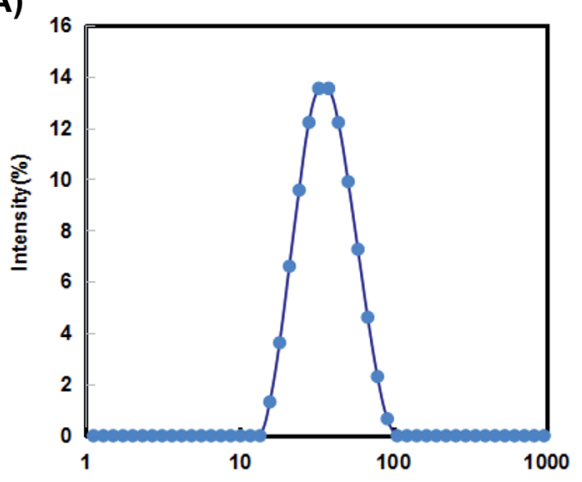

C)

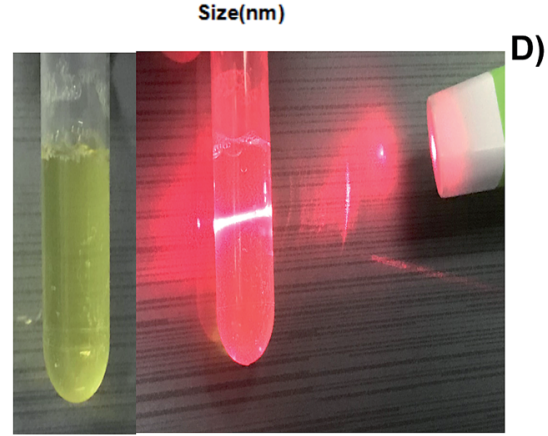

D)
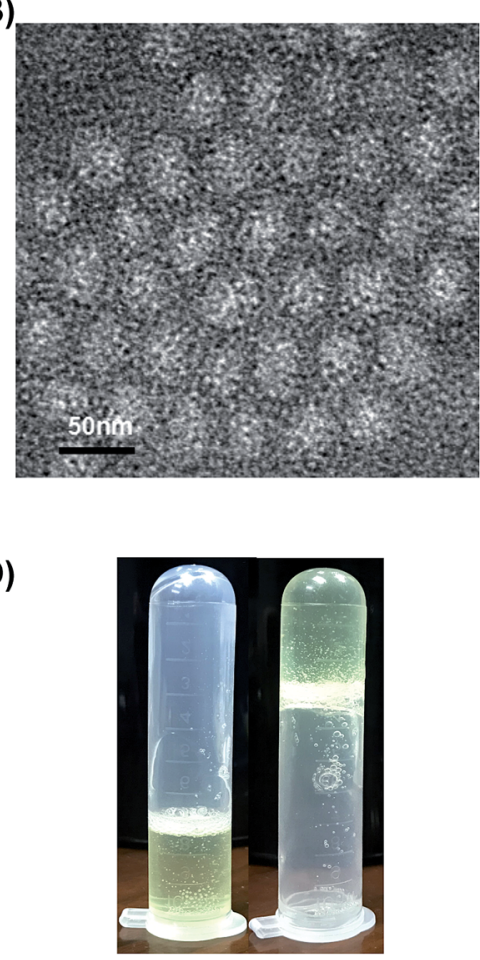

E)

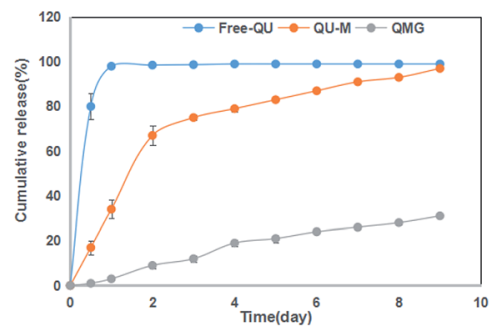

Fig. 2 Characterization of Qu-M and the Qu-M-hydrogel composites. (A) The size distribution of Qu-M (QM), (B) TEM image of Qu-M (QM), (C) photos of the clear and homogeneous Qu-M-hydrogel composites (QMGs) and the Tyndall effect (from left to right), (D) photos of the Qu-Mhydrogel composites (QMGs) with liquid state and hydrogel state at temperatures of $4{ }^{\circ} \mathrm{C}$ and $37^{\circ} \mathrm{C}$ (from left to right), and (E) in vitro release study. 
into the biodegradable nano-sized amphiphilic block copolymers of MPEG-PCL with the self-assembly method mentioned in Fig. 1. As shown in Fig. 1, Qu-M has a core-shell structure, which is composed of a hydrophobic inner core of PCL and a hydrophilic outer shell of PEG, and quercetin was enwrapped in the PCL core. Then, the Qu-nano-micelles (QM) were added to the hydrogel at $4{ }^{\circ} \mathrm{C}$, and dispersed to form a hydrogel.

3.1.2 Characterization of Qu-M and the Qu-M-hydrogel composites. According to high performance liquid chromatography (HPLC) and the above formulae (1) and (2), the drug loading (DL) and entrapment efficiency (EE) of Qu-M (QM) were $6.85 \pm 0.12 \%$ and $97.8 \pm 0.34 \%$, respectively, indicating that the method was desirable and the quality of the preparation was good.

The particle size and zeta potential of Qu-M (QM) were measured in detail through dynamic light scattering (DLS) and transmission electron microscopy (TEM). As shown in Fig. 2A, the particle size of Qu-M (QM) in liquid phase analysed by DLS was $36.0 \pm 3.2 \mathrm{~nm}$ with a polydispersity index (PDI) of $0.14 \pm 0.08$, suggesting that Qu-M (QM) had a narrow particle size distribution with uniform size. The zeta potential of Qu-M (QM) is $-1.56 \pm 0.15 \mathrm{mV}$ (date not shown), indicating that the micellar dispersions have good stability.

Observed by TEM, in the solid phase (Fig. 2B), the Qu-M composites (QM) had a spherical structure, with an average diameter of about $20 \mathrm{~nm}$, shorter than in the liquid phase, which was due to the relatively loose structure of the amphiphilic block copolymer (MPEG-PCL) and hydrogel in liquid phase.

Quercetin enveloped by polymeric micelles of MPEG-PCL and hydrogel had better water-solubility than the free one, as shown in Fig. 2C. It is visible that the nanometer preparation solution was clear and homogeneous in appearance, which confirmed that the quercetin low water solubility problems were improved significantly.

One of the main purposes of packaging quercetin with MPEG-PCL micelles was to make quercetin be completely dispersed in aqueous solution. As can be seen from Fig. 2D, the Qu-M-hydrogel composite (QMG) solution is flowing liquid at $4{ }^{\circ} \mathrm{C}$ but changed to gel state at $37{ }^{\circ} \mathrm{C}$ indicating that QMG could change to a gel in vivo, and quercetin was released from QMG over a prolonged period compared with free-Qu and Qu-M.

\subsection{In vitro anti-tumor activity}

3.2.1 Qu-M inhibited ovarian cancer cell growth in vitro more effectively. The cytotoxic effects of free quercetin (FQ) and Qu-M (QM) on ovarian cancer SKOV-3 cells in vitro were evaluated by the cell viability assay. As depicted in Fig. 3A and B, free quercetin (FQ) and Qu-M (QM) inhibited the growth of the SKOV-3 cells significantly in vitro in a concentration-dependent and time-dependent manner. The semi-inhibitory concentration (IC50) of the free quercetin at 48 hours was $16.21 \mu \mathrm{g} \mathrm{mL}^{-1}$, inferior to that of QM $\left(13.87 \mu \mathrm{g} \mathrm{mL}^{-1}\right)$, demonstrating that the
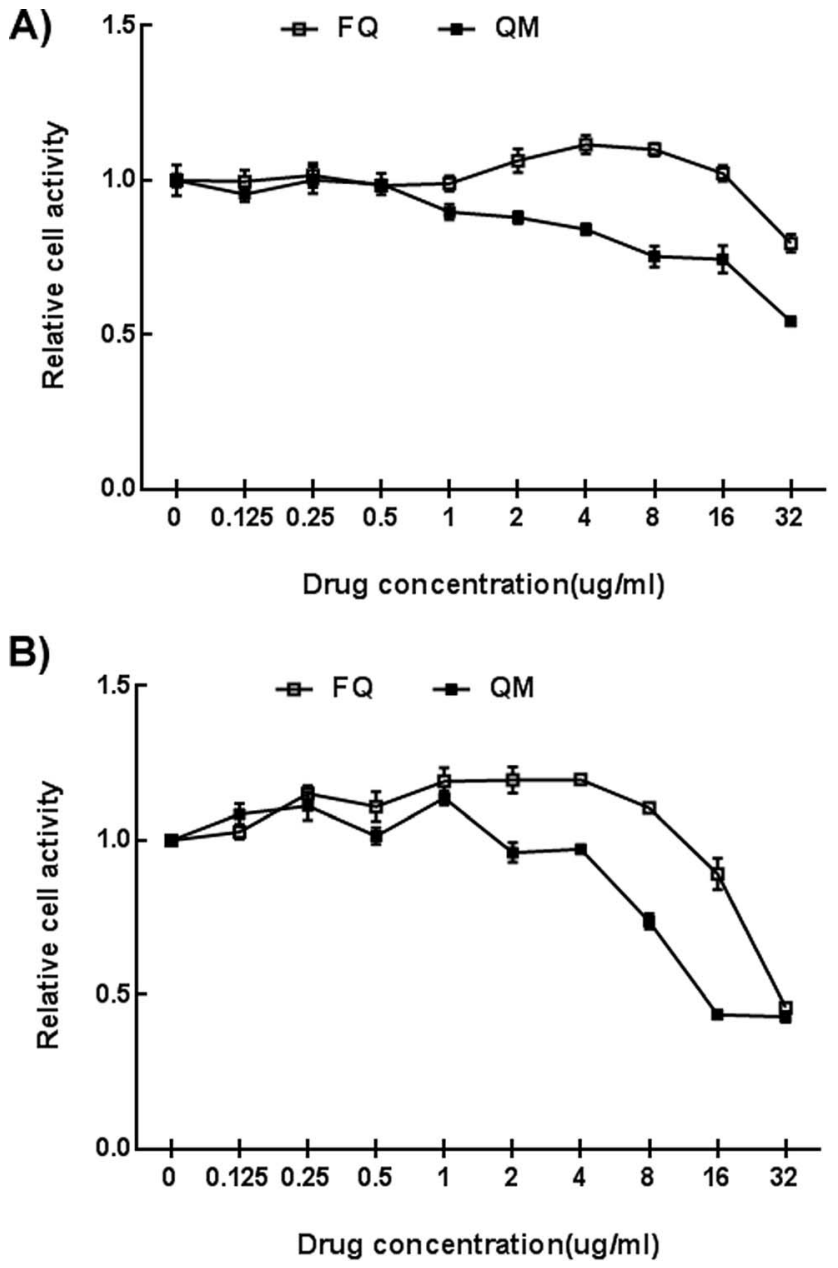

Fig. 3 The cytotoxicity of Qu-M and free-Qu on the SKOV-3 cells in vitro. Free-Qu (FQ), as well as Qu-M (QM) could efficiently inhibit the viability of the SKOV-3 cells in a dose-dependent manner after 24 hours (A) and 48 hours (B). Also, Qu-M (QM) could enhance the cytotoxic activity of quercetin on the SKOV-3 cells in vitro.

drug-loaded block copolymer (MPEG-PCL) could elevate the cytotoxicity of quercetin in vitro.

3.2.2 Qu-M induced ovarian cancer cell apoptosis. The apoptosis effects of Qu-M (QM) on the ovarian cancer SKOV-3 cells in vitro were assessed by flow cytometry. As illustrated in Fig. 4, the apoptotic rates of the early and late cells in different six subgroups (control and drug concentrations of $64 \mu \mathrm{g} \mathrm{mL} \mathrm{m}^{-1}$, $32 \mu \mathrm{g} \mathrm{mL}{ }^{-1}, 16 \mu \mathrm{g} \mathrm{mL}{ }^{-1}, 8 \mu \mathrm{g} \mathrm{mL}^{-1}$, and $4 \mu \mathrm{g} \mathrm{mL}^{-1}$ ) were $3.65 \%$ $\pm 0.71 \%, 76.43 \% \pm 3.62 \%, 33.95 \% \pm 1.62 \%, 14.44 \% \pm 1.41 \%$, $11.46 \% \pm 1.21 \%$, and $7.03 \% \pm 1.06 \%$, respectively. The results showed that the apoptosis-inducing effect of QM on the SKOV-3 cells in vitro was concentration-dependent.

\subsection{In vivo anti-tumor activity}

3.3.1 Qu-M and the Qu-M-hydrogel composites inhibited ovarian cancer more effectively in vivo. In order to assess the anti-tumor effect of the free quercetin (FQ), Qu-M (QM), and Qu$\mathrm{M}$-hydrogel composites (QMGs) on ovarian cancer in vivo, the abdominal SKOV-3 cancer mouse model was established. 

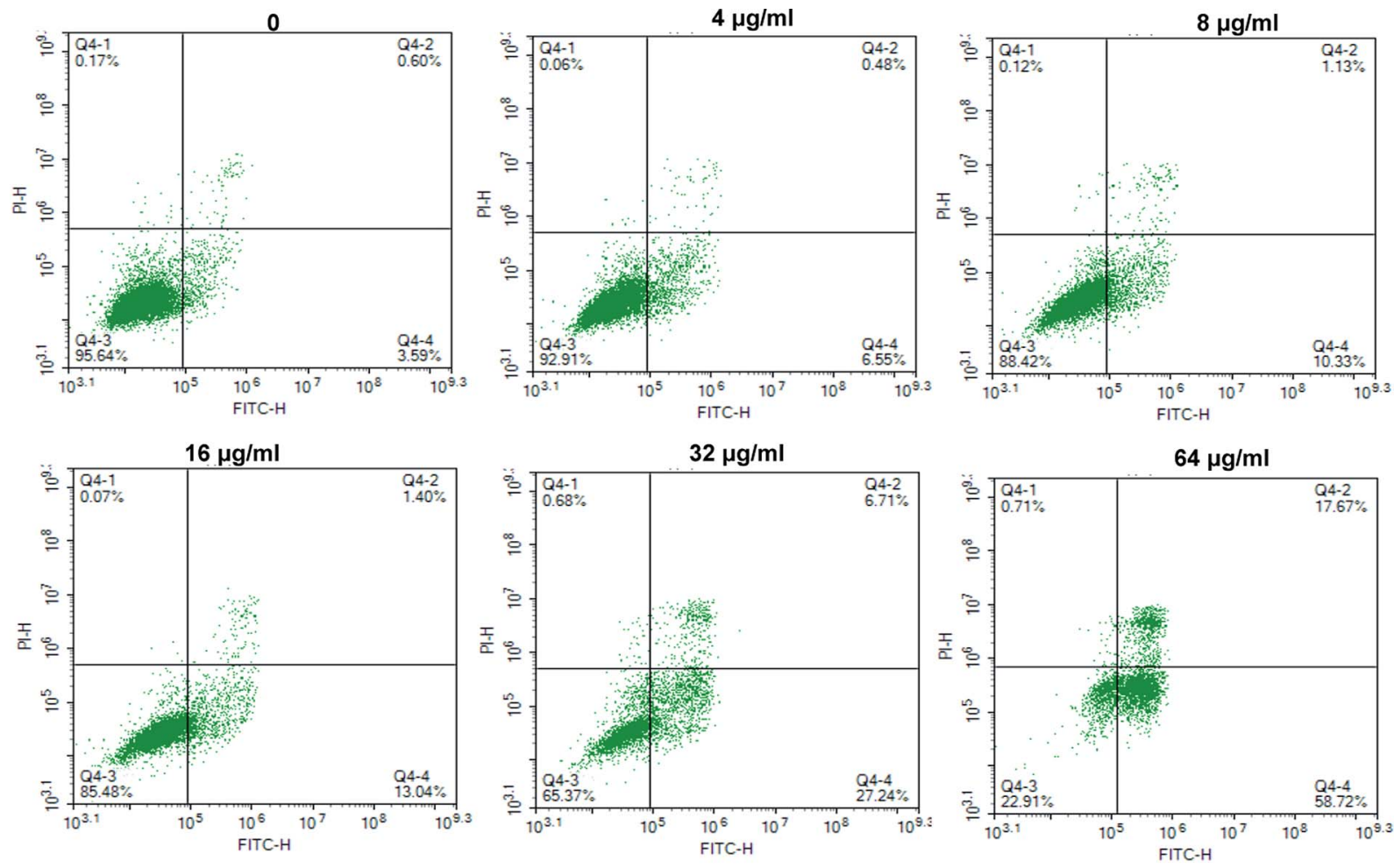

Fig. 4 The apoptosis effects of Qu-M in a dose-dependent manner on the SKOV-3 cells in vitro. The SKOV-3 cells were incubated with Qu-M (QM) for 48 hours, and the Annexin V and PI-stained cells were determined by Flow Cytometry (FCM).

A)
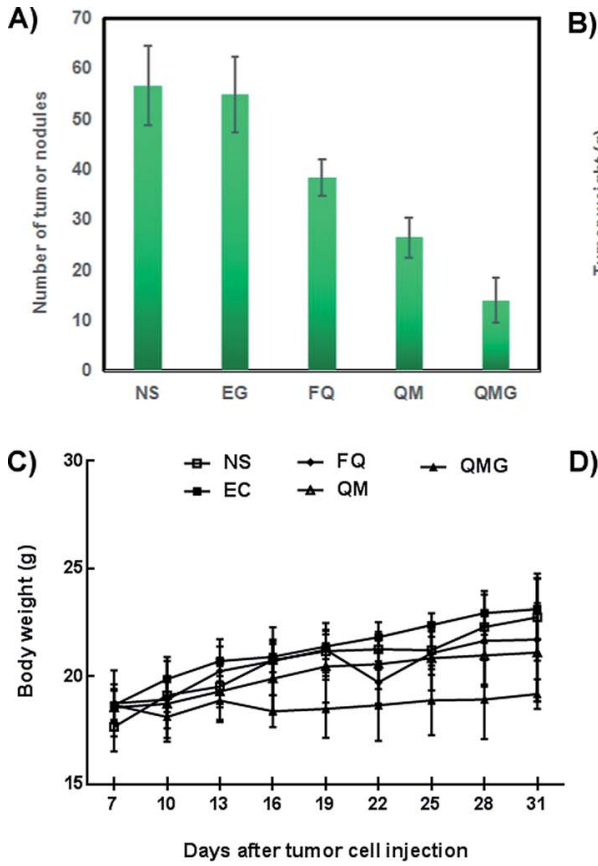

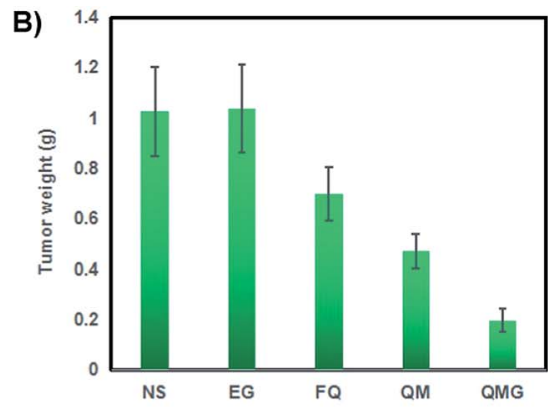

D)

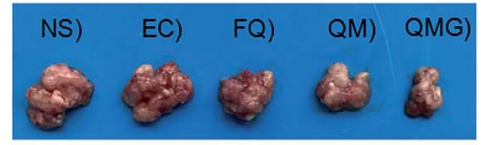

Fig. 5 The therapy efficiency of free-Qu, Qu-M and the Qu-M-hydrogel composites on the mice subcutaneous SKOV-3 cancer model in vivo. (A) The number of tumor nodules on the harvested day. (B) The tumor weights measured on the harvested day. (C) The body weights of the mice measured on the indicated days. (D) Representative images of the tumor gross specimens in each treated group, indicating that the Qu-Mhydrogel composites (QMGs) were more efficacious in repressing the growth of the tumor in vivo. 

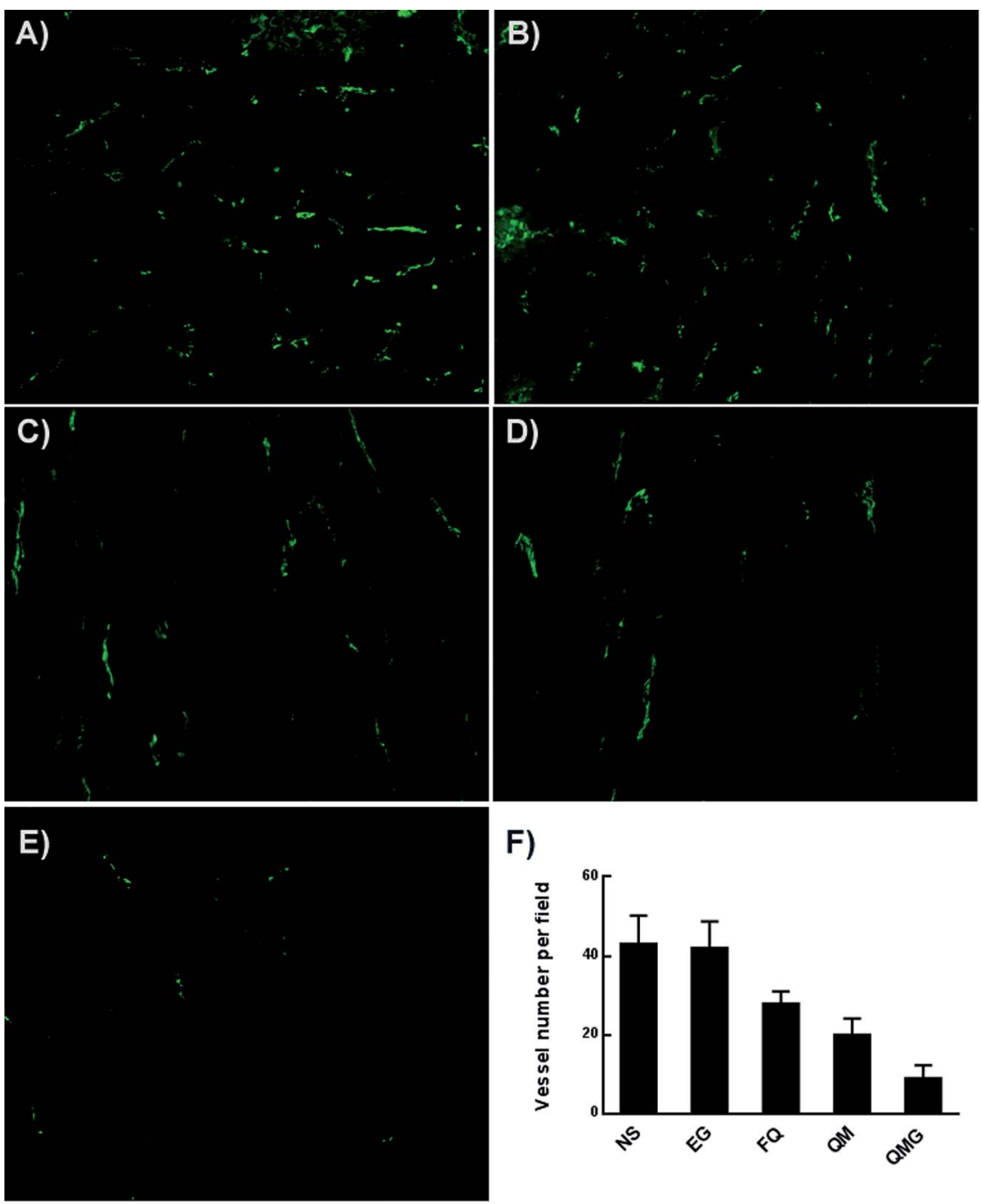

F)

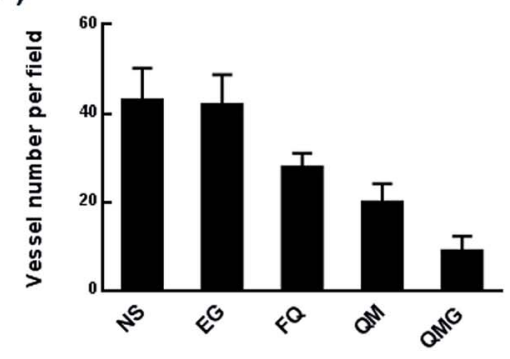

Fig. 6 CD31 assay. The tumor tissue sections of the normal saline (NS) treated group (A), empty hydrogel (EG) treated group (B), free quercetin (FFQ) treated group (C), Qu-M (QM) treated group (D) and Qu-M-hydrogel composite (QMG) treated group (E) were immunostained with CD31 for evaluating the microvessel density (F), implying that anti-angiogenesis may be another anti-tumor mechanism of the Qu-M-hydrogel composites (QMGs), Qu-M (QM), and free quercetin (FQ) in vivo.

According to Fig. 5A and B, in the Qu-M-hydrogel composites (QMGs), Qu-M (QM), free quercetin (FQ), empty hydrogel (EG), and normal saline (NS) groups, the number of tumor nodules was significantly increased to $14 \pm 4.5,26.4 \pm 4.04$, $38.4 \pm 3.65,54.8 \pm 7.5$ and $56.6 \pm 7.83$, respectively, and the tumor weights were $0.198 \pm 0.049 \mathrm{~g}, 0.47 \pm 0.068 \mathrm{~g}, 0.7 \pm$ $0.107 \mathrm{~g}, 1.038 \pm 0.173 \mathrm{~g}$, and $1.028 \pm 0.177 \mathrm{~g}(P<0.05)$. As is shown in Fig. 5C, there was no significant change in body weight of each treatment group. The results of the above animal experiments demonstrated that empty hydrogel (EG) had no obvious anti-tumor biological activity against ovarian cancer, whereas quercetin had significant tumor growth inhibitory effect, especially in the form of Qu-M-hydrogel composites (QMGs), without having an effect on the body weight. As shown in Fig. 5D, the pictures of representative individuals of all the groups were convincing proof of the therapy effect.
3.3.2 Anti-angiogenesis in vivo. The immunofluorescence analysis of neovascularization was used to detect the antiangiogenesis activities of free quercetin (FQ), Qu-M (QM), and the Qu-M-hydrogel composites (QMGs) in the tumor tissues of animal models. As is shown in Fig. 6, the tumor microvessels show green fluorescence in different densities. The microvessel density (MVD) in the Qu-M-hydrogel composites (QMGs), Qu-M (QM) and free quercetin (FQ), empty hydrogel (EG), and normal saline (NS) groups were $9 \pm 3.3,20 \pm 4.2,28 \pm 3.1,42 \pm 6.7$ and $43 \pm 7.2$, respectively, suggesting that the inhibition of tumor microvascularization may be another mechanism of quercetin in the anti-ovarian tumor effect.

3.3.3 Anti-proliferation in vivo. The experimental data and Fig. 7 show a large number of proliferating cells in the tissue sections showing green fluorescence in the empty hydrogel (EG) and normal saline (NS) treated groups, with cell proliferation rates of $89.3 \% \pm 15.63 \%$ and $90.3 \% \pm 11.67 \%$, respectively, 


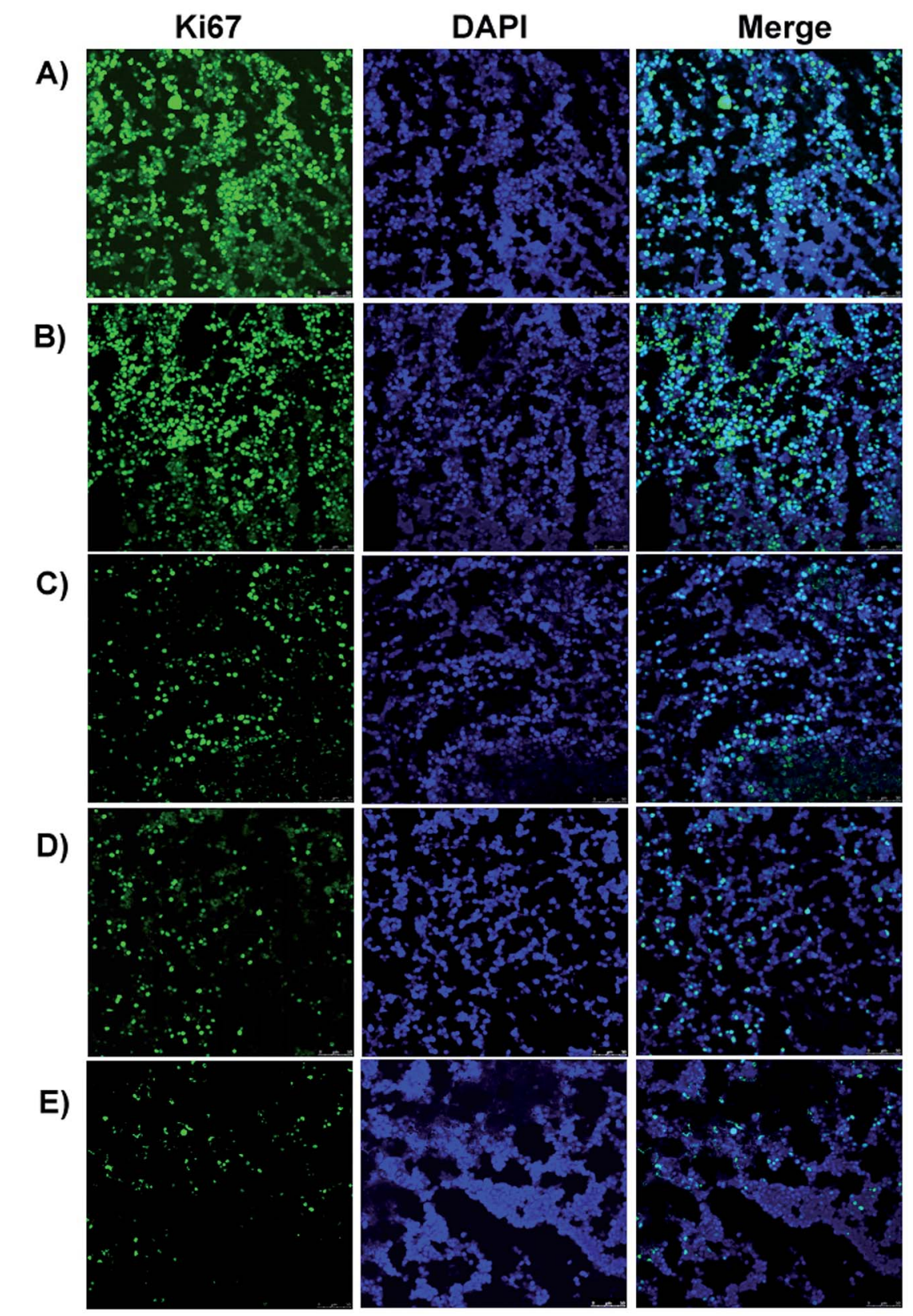

Fig. 7 Ki-67 assay. The tumor tissue sections of the normal saline (NS) treated group (A), empty hydrogel (EG) treated group (B), free quercetin $(F Q)$ treated group (C), Qu-M (QM) treated group (D), and Qu-M-hydrogel composite (QMG) treated group (E) were immunostained with Ki-67 for evaluating the cell proliferation, indicating that anti-proliferation may be the other anti-tumor mechanism of the Qu-M-hydrogel composites (QMGs), Qu-M (QM), and free quercetin (FQ) in vivo.

indicating that the empty hydrogel (EG) had no obvious inhibition of cell proliferation on SKOV-3 cells. Whereas, groups of free quercetin (FQ), Qu-M (QM) and the Qu-M-hydrogel composites (QMGs) revealed pronounced anti-proliferation effects with lower data of $56.3 \% \pm 9.63 \%, 46.3 \% \pm 9.63 \%$, and $17.45 \% \pm 4.64 \%$, respectively, showing that antiproliferation effects may be another one of the anti-tumor mechanisms of quercetin.

3.3.4 Induction of tumor cell apoptosis in vivo. In Fig. 8, it was obvious that more apoptotic cells showing green fluorescence could be seen in picture $\mathrm{E}$ than in the other four pictures.
The apoptotic indexes in the Qu-M-hydrogel composites (QMGs), Qu-M (QM), free quercetin (FQ), empty hydrogel (EG) and normal saline (NS) were $72.7 \% \pm 6.34 \%, 43.23 \% \pm 4.68 \%$, $28.23 \% \pm 3.23 \%, 2.14 \% \pm 0.57 \%$, and $1.31 \% \pm 0.43 \%$, respectively, indicating that quercetin in the form of the Qu-Mhydrogel composites (QMGs) had stronger effect on tumor cell apoptosis, and at the same time, revealing that induction of tumor cell apoptosis may be an anti-tumor mechanism of quercetin in vivo.

3.3.5 In vivo toxicity study. In vivo studies showed that the Qu-M-hydrogel composites (QMGs), Qu-M (QM), free quercetin 

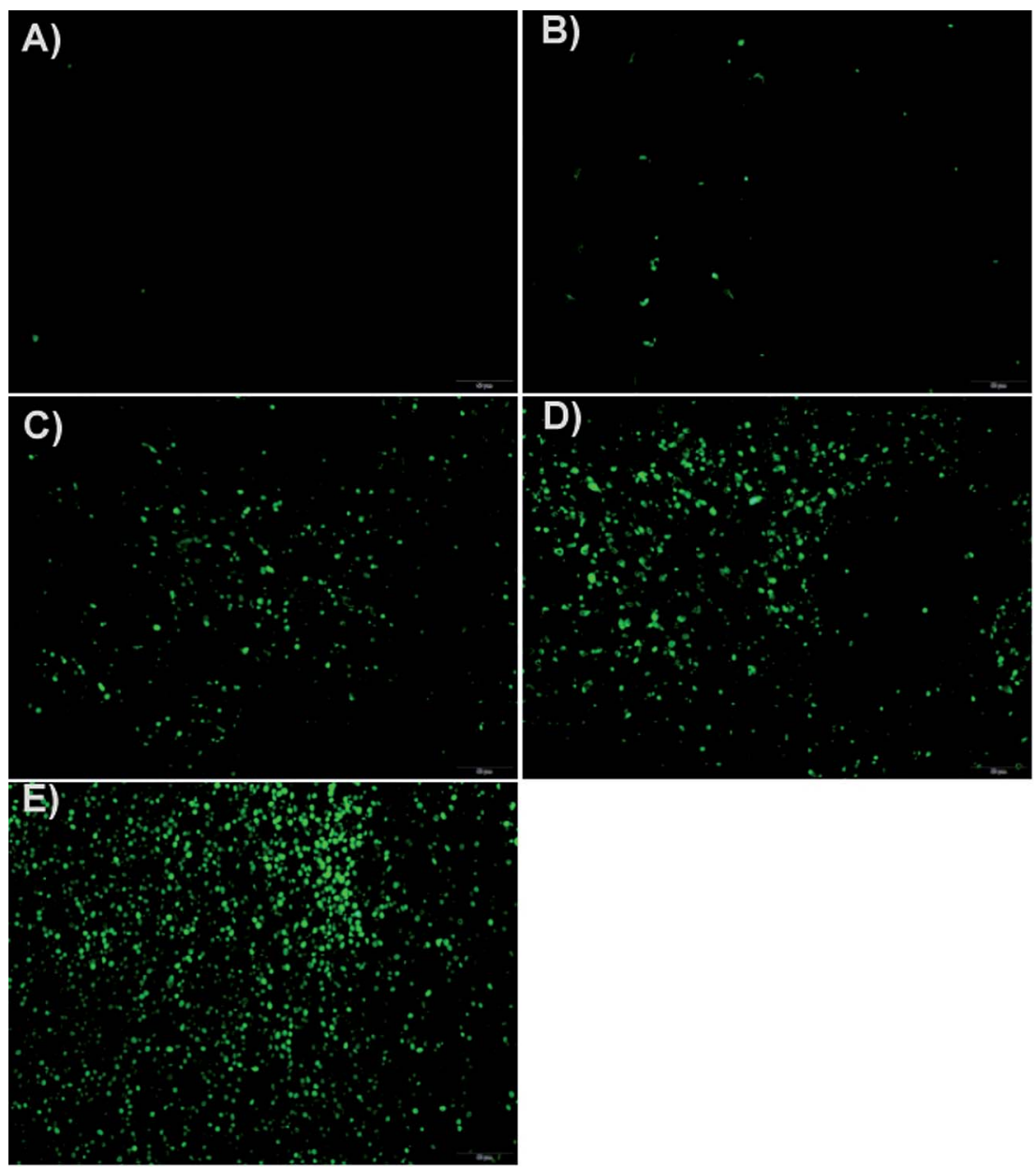

Fig. 8 Tunnel assay. The tumor tissue sections of the normal saline (NS) treated group (A), empty hydrogel (EG) treated group (B), free quercetin $(F Q)$ treated group (C), Qu-M (QM) treated group (D), and Qu-M-hydrogel composite (QMG) treated group (E) were stained with Tunnel for the cell apoptosis assay, indicating that inducing apoptosis may be one of the anti-tumor mechanisms of the Qu-M-hydrogel composites (QMGs), Qu-M (QM), and free quercetin (FQ) in vivo.

(FQ), empty hydrogel (EG), and normal saline (NS) did not change the histology and serum biochemical and hematology parameters of the main organs (heart, liver, lung, kidney and spleen).

The histological analysis of the main organs showed no significant changes between the treatment groups and nontreatment group (Fig. 9). There were no signs of necrosis, regeneration changes, and cell replacement, and normal parenchymal cells and organ structures were observed.

Several serum biochemical parameters, including functional markers, such as albumin (ALB), alkaline phosphatase (ALP), alanine aminotransferase (ALT), aspartate aminotransferase (AST), blood urea nitrogen (BUN), cholesterol, creatine kinase (CK), creatinine (CREA), glucose (GLU), high density lipoprotein (HDL), lactate dehydrogenase (LDH), low density lipoprotein (LDL), total bilirubin (TBIL) triglycerides (TG), total proteins (TP), uric acid (UA) and XAMY were also determined (Fig. 10). We found that all biochemical parameters determined after treatments were within the normal ranges and were statistically comparable to the control group.
The hematological parameters, including white blood cell count (WBC), red blood count (RBC), hemoglobin (HGB), and platelet (PLT), were monitored to assess the eventual hematology impairment due to the administration of the developed nanoparticles (Fig. 11). In general, no statistically significant toxicities in the hematology parameters were found after treatments.

Together, the results from the in vivo toxicity study suggest a lack of signs of toxicity and good biocompatibility of the QuM-hydrogel composites (QMGs), Qu-M (QM), free quercetin (FQ), empty hydrogel (EG), and normal saline (NS).

\section{Discussions}

At present, chemotherapy is one of the primary effective methods to treat cancers, among surgery, radiation, and biological therapy. However, the traditional chemical treatment is a double-edged sword, due to having more or less side effects on the normal tissue and organs due to the drugs' cytotoxicity. In order to minimize and even avoid the toxic side effects, safe and 


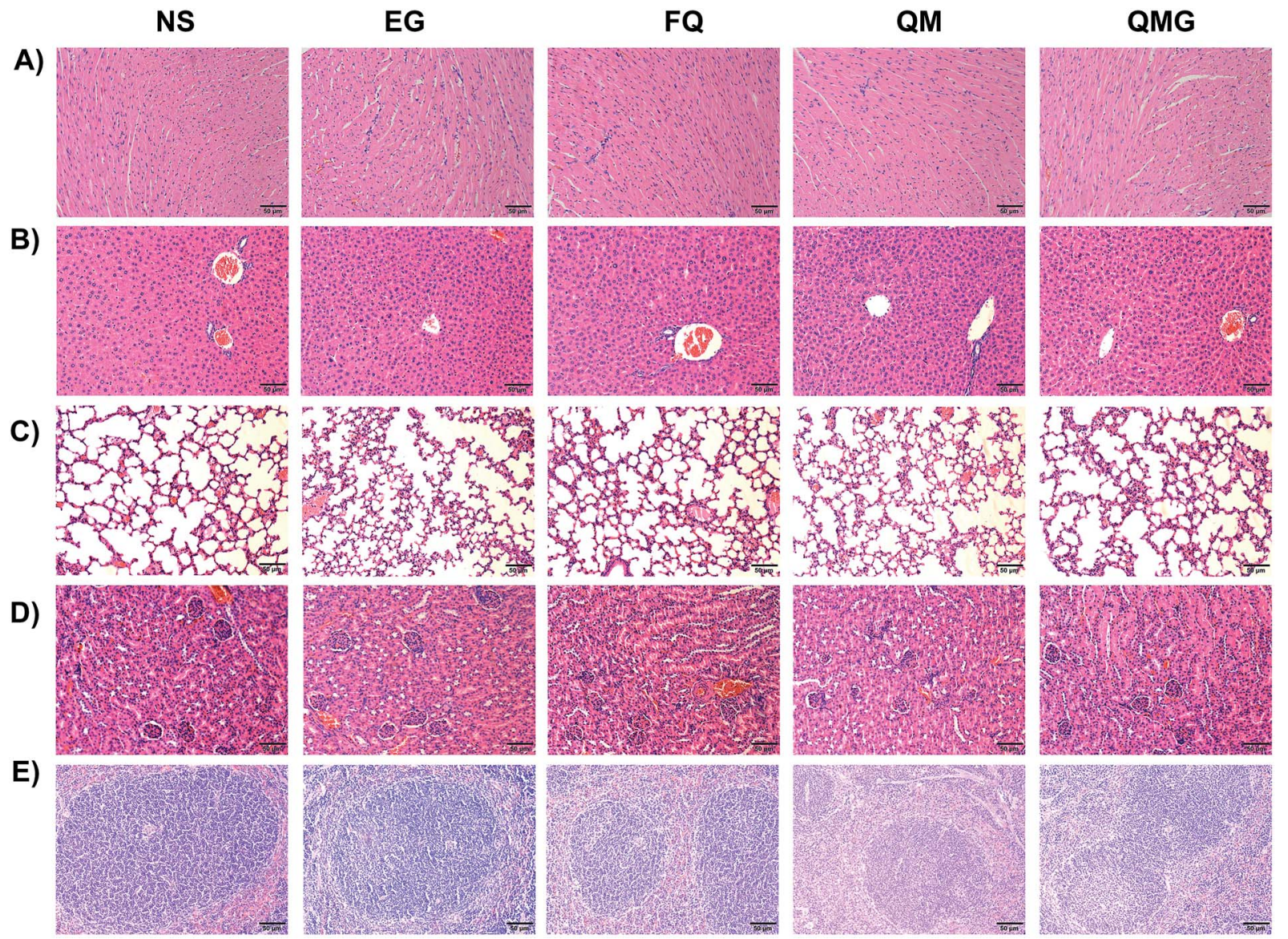

Fig. 9 The histological analysis with HE staining. The histological analysis of the heart (A), liver (B), lung (C), kidney (D) and spleen (E) organs. The main organ tissue sections stained with $\mathrm{H} \& \mathrm{E}$ showed no significant changes between the treatment groups of normal saline (NS), empty hydrogel (EG), free quercetin (FQ), Qu-M (QM) and the $Q u-M$-hydrogel composites (QMGs).

effective natural phytochemicals have become a hot spot for drug research and development. ${ }^{41-43}$ Quercetin, as a natural flavonoid compound, with its wide range of pharmacological effects, has aroused the keen interests of researchers. Its prominent advantage is that it is easy to get from daily foods, such as vegetables and fruits. Recent studies have shown that quercetin has anti-arrhythmic, anti-proliferation, and antitumor activities, resulting in its potential application in tumor chemotherapy.

Although quercetin has a broad medicinal prospect, its low water solubility has greatly limited its use in clinical therapy. Furthermore, quercetin, delivered by injection, will be rapidly metabolized and cleared in the body, which reduces its bioavailability to a certain extent. Therefore, how to effectively solve the problem of low hydrophilicity of quercetin, prolong the cycle time in vivo, and improve its bioavailability has become the focus of scientific research. It is encouraging that with the advancement of molecular science and nanotechnology, the nano-drug delivery system provides an effective platform for support. ${ }^{44-46}$ It has provided an important approach for water-soluble preparation of hydrophobic drugs, targeting, and releasing slowly, which has become a research direction at home and abroad. ${ }^{47,48}$
The types of nano-drug-loaded systems can be subdivided into nano-liposomes, solid lipid nanoparticles, nanocapsules/nanospheres, and polymer micelles, in which polymer micelles are a nano-carrier newly developed in recent years. ${ }^{49,50}$ Polymer micelles are assembled by biodegradable amphiphilic blocks or graft copolymers, which are both hydrophilic and hydrophobic, suitable for carrying different properties of the drugs. Hydrophobic drugs encapsulated in polymer micelles can be completely dissolved in aqueous solution to form a stable, homogeneous nanoparticle formulation. ${ }^{51}$

In addition, the tiny size and stable hydrophilic interface of the polymer micelles can extend the time of drug release and circulation in vivo, thereby increasing the cellular uptake. More importantly, polymer micelles can target tumors through the high permeability and retention (EPR) effect, thereby improving the anti-tumor efficacy. ${ }^{52}$ Polyethylene glycol monomethyl ether-polycaprolactone block copolymer (MPEG-PCL) has the advantages of easy preparation and biodegradability, and has attracted wide attention as a drug delivery carrier. ${ }^{53,54}$

In this study, MPEG-PCL was used as a drug delivery vehicle to encapsulate quercetin via self-assembly into a capsule. Then 

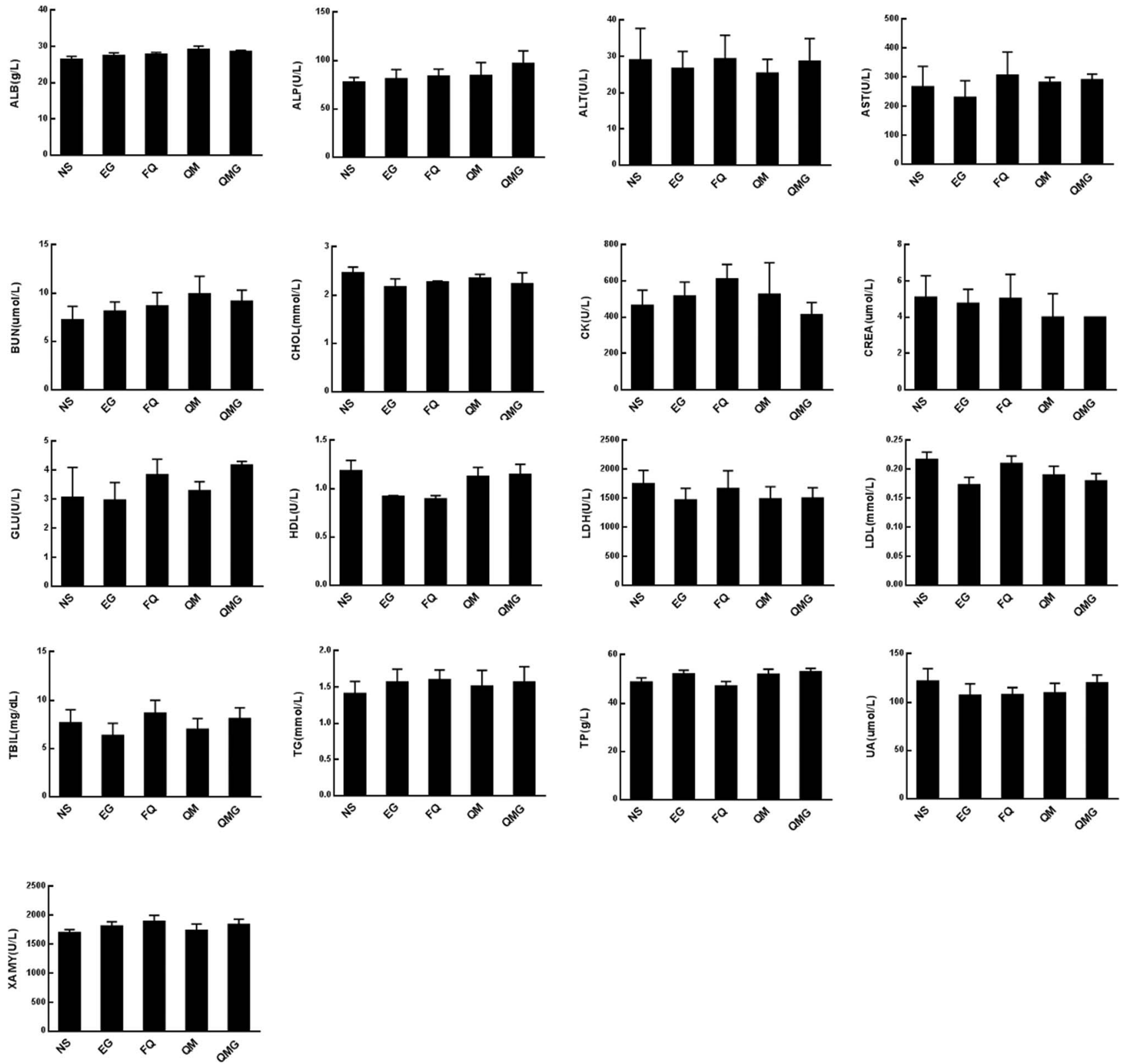

Fig. 10 Serum biochemical parameter analysis. The analysis of the serum biochemical parameters including albumin (ALB), alkaline phosphatase (ALP), alanine aminotransferase (ALT), aspartate aminotransferase (AST), blood urea nitrogen (BUN), cholesterol, creatine kinase (CK), etc. All the biochemical parameters determined after treatments were within the normal ranges and were statistically comparable to the control group.

the capsule was loaded into thermosensitive hydrogel to form homogeneous Qu-M-hydrogel composites. The obtained QuM-hydrogel composites, with uniform size, high capacity, and high drug encapsulation efficiency, can be dispersed in aqueous solution homogeneously without metamorphism. The Qu-Mhydrogel composite performed better than free quercetin and Qu-M in the cytotoxicity apoptosis inducing effect on the SKOV3 cells in vitro.

In addition, the subcutaneous SKOV-3 ovarian cancer mouse model was established, in which the Qu-M-hydrogel composites had more marked inhibitory effect on the tumor growth than free quercetin and Qu-M. TUNEL assay, CD31 assay, and
Ki67 immunofluorescence assay revealed that quercetin had significant biological activity of induction of tumor cell apoptosis, inhibition of tumor angiogenesis, and cell proliferation in vivo, suggesting that the three may be the essential antiovarian cancer mechanism of quercetin.

Seen from the data of the experiments, the Qu-M-hydrogel composites show better anti-cancer effect than free quercetin and Qu-M in vitro and in vivo, which objectively confirmed that the Qu-M-hydrogel composites effectively improved the low water solubility of quercetin and its bioavailability, suggesting that in the clinical treatment of ovarian cancer, quercetin nano preparations have potential application prospects. 

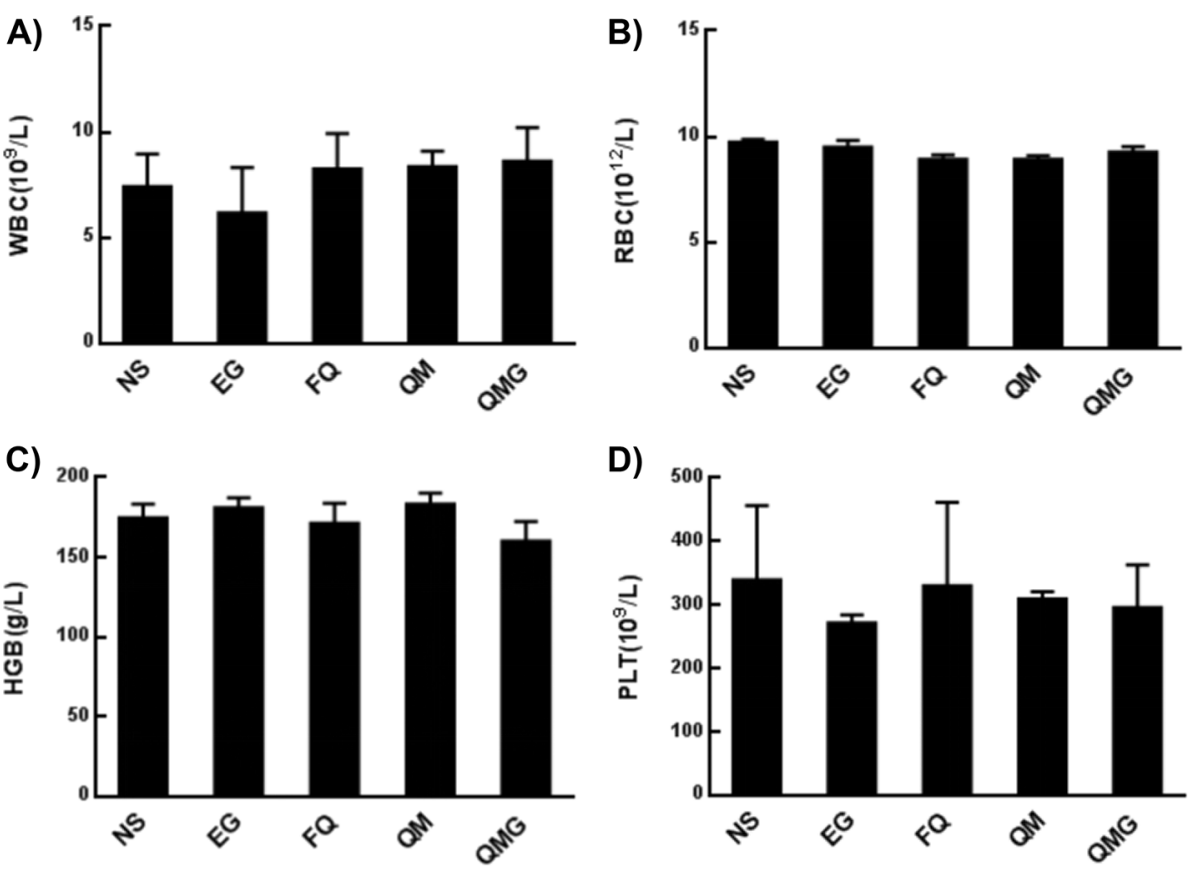

Fig. 11 Hematological parameters analysis. The analysis of the hematological parameters including white blood cell count (A), red blood count $(B)$, hemoglobin (C), and platelet (D). There is no statistically significant toxicities in the hematology parameters found after the treatments of normal saline (NS), empty hydrogel (EG), free quercetin (FQ), Qu-M (QM), and the Qu-M-hydrogel (QMGs) composites.

\section{Conclusions}

In summary, a quercetin-loaded drug delivery system was successfully designed by combining the polyethylene MPEGPCL and thermosensitive hydrogel and was tested for its characterization and anti-tumor effects on ovarian cancer. The results obtained showed that quercetin in the form of the QuM-hydrogel composites demonstrated excellent anti-cancer activities. The thermosensitive hydrogel shell of the structure could keep quercetin release steady and sustained without bursting, thereby enhancing drug efficacy, and the data of more cancer cell apoptosis, inhibition of angiogenesis, and cell proliferation proved the function of the new micelle structure. All the results show that the prepared Qu-M-hydrogel composites may have potential applications in ovarian cancer chemotherapy.

\section{Conflicts of interest}

The authors report no conflicts of interest in this work.

\section{References}

1 R. L. Siegiel, K. D. Miller and A. Jemal, Ca-Cancer J. Clin., 2017, 67(1), 7-30.

2 P. M. Webb and S. J. Jordan, Best Pract. Res. Clin. Obstet. Gynaecol., 2017, 41, 3-14.

3 Y. Miao, Q. Yan, S. Li, et al., Cancer Biomarkers, 2016, 17(1), 33-40.

4 G. Jiang, J. Liu, B. Ren, et al., J. Ethnopharmacol., 2016, 193, 368-376.
5 G. C. Jayson, E. C. Kohn, H. C. Kitchener, et al., Lancet, 2014, 384(9951), 1376-1388.

6 L. C. Woodhouse and R. J. Edmondson, International Encyclopedia of Public Health, 2nd edn, 2017, pp. 378-382.

7 J. García-Pérez, V. Lope, G. López-Abente, et al., Environ. Pollut., 2015, 205, 103-110.

8 S. Xu, H. Fan, L. Yin, et al., Eur. J. Pharm. Biopharm., 2016, 104, 251-259.

9 E. P. Strandmann, S. Reinartz, U. Wager, et al., Trends in Cancer, 2017, 3(2), 137-148.

10 Q. Wu, S. Deng, L. Li, et al., Nanoscale, 2013, 5(24), 1248012493.

11 M. Lou, L. Zhang, P. Ji, et al., Biomed. Pharmacother., 2016, 84, 1-9.

12 Y. R. Liao and J. Y. Lin, Life Sci., 2015, 137, 89-97.

13 W. Wang, C. Sun, L. Mao, et al., Trends Food Sci. Technol., 2016, 56, 21-38.

14 G. Xu, H. Shi, L. Ren, et al., Int. J. Nanomed., 2015, 10, 20512063.

15 A. Cassidy, T. Huang, M. S. Rice, et al., Am. J. Clin. Nutr., 2014, 100(5), 1344-1351.

16 L. Yi, Y. Zongyuan, G. Cheng, et al., Cancer Sci., 2014, 105(5), 520-527.

17 N. Li, C. Sun, B. Zhou, et al., PLoS One, 2014, 9(7), e100314.

18 Z. Yang, Y. Liu, J. Liao, et al., FEBS J., 2015, 282(6), 11111125.

19 Y. Liu, W. Gong, Z. Y. Yang, et al., Apoptosis, 2017, 22(4), 544557.

20 M. X. Ren, X. H. Deng, F. Ai, et al., Exp. Ther. Med., 2015, 10(2), 579-583. 
21 J. Zhou, J. Gong, C. Ding, et al., Mol. Med. Rep., 2015, 12(2), 3127-3131.

22 A. Parvaresh, R. Razavi, N. Rafie, et al., J. Res. Med. Sci., 2016, 21, 195-201.

23 Q. Long, Y. Xie, Y. Huang, et al., J. Biomed. Nanotechnol., 2013, 9(6), 965-975.

24 A. Filipa Brito, M. Ribeiro, A. Margarida Abrantes, et al., Curr. Med. Chem., 2015, 22(26), 3025-3039.

25 T. Iwamoto, Biol. Pharm. Bull., 2013, 36(5), 715-718.

26 N. Muhammad and Z. Guo, Curr. Opin. Chem. Biol., 2014, 19, 144-153.

27 W. Song, Z. Tang, M. Li, et al., Acta Biomater., 2014, 10(3), 1392-1402.

28 E. Pérez-Herrero and A. Fernández-Medarde, Eur. J. Pharm. Biopharm., 2015, 93, 52-79.

29 E. Hall, D. Cameron, R. Waters, et al., Eur. J. Cancer, 2014, 50(14), 2375-2389.

30 Y. Q. Du, X. X. Yang, W. L. Li, et al., RSC Adv., 2014, 4(66), 34830-34835.

31 R. Tian, J. Chen and R. Niu, Nanoscale, 2014, 6(7), 34743482.

32 M. Norouzi, B. Nazari and D. W. Miller, Drug Discovery Today, 2016, 21(11), 1835-1849.

33 G. R. Dakwar, M. Shariati, W. Willaert, et al., Adv. Drug Delivery Rev., 2017, 108, 13-24.

34 W. Solass, R. Kerb, T. Mürdter, et al., Ann. Surg Oncol., 2014, 21(2), 553-559.

35 D. S. Kohane, J. Y. Tse, Y. Yeo, et al., J. Biomed. Mater. Res., Part A, 2006, 77(2), 351-361.

36 S. Dadashzadeh, N. Mirahmadi, M. H. Babaei, et al., J. Controlled Release, 2010, 148(2), 177-186.

37 K. Park, J. Controlled Release, 2012, 158(3), 355.
38 P. Huang, H. Song, Y. Zhang, et al., ACS Appl. Mater. Interfaces, 2016, 8(43), 29323-29333.

39 L. Klouda, Eur. J. Pharm. Biopharm., 2015, 97, 338-349.

40 G. Deepa, A. K. T. Thulasidasan, R. J. Anto, et al., Int. J. Nanomed., 2012, 7, 4077.

41 T. N. Chinembiri, L. H. Du Plessis, M. Gerber, et al., Molecules, 2014, 19(8), 11679-11721.

42 A. Chen, J. Xu and A. C. Johnson, Oncogene, 2006, 25(2), 278287.

43 G. O’Sullivan-Coyne, G. C. O'sullivan, T. R. O'Donovan, et al., Br. J. Cancer, 2009, 101(9), 1585-1595.

44 X. Li, Z. Zhang, J. Li, et al., Nanoscale, 2012, 4(15), 4667-4673. 45 M. C. Parrott and J. M. DeSimone, Nat. Chem., 2012, 4(1), 1314.

46 G. Guo, S. Z. Fu, L. X. Zhou, et al., Nanoscale, 2011, 3(9), 3825-3832.

47 Q. Wang, X. Zhuang, J. Mu, et al., Nat. Commun., 2013, 4, 1867.

48 C. J. Brinker, Nat. Mater., 2012, 11(10), 831-832.

49 X. Gao, B. L. Wang, X. W. Wei, et al., Nanoscale, 2012, 4(22), 7021-7030.

50 H. Cabral, Y. Matsumoto, K. Mizuno, et al., Nat. Nanotechnol., 2011, 6(12), 815-823.

51 C. Gong, Y. Xie, Q. Wu, et al., Nanoscale, 2012, 4(19), 60046017.

52 C. Y. Gong, C. Wang, Y. J. Wang, et al., Nanoscale, 2012, 4(10), 3095-3104.

53 M. L. Gou, X. W. Wei, K. Men, et al., Curr. Drug Targets, 2011, 12(8), 1131-1150.

54 S. Chakraborty, S. Stalin, N. Das, et al., Biomaterials, 2012, 33(10), 2991-3001. 